\title{
The Signal-Averaged Electrocardiogram of $P$ Wave in Patients with Documented Atrial Fibrillation or Flutter and in Patients with Left or Right Atrial Overload without Atrial Fibrillation
}

\author{
Hidemi Ogawa, M.D., Tomoo Inoue, M.D., \\ Akihiro Yoshida, M.D., Tomofumi Dor, M.D., \\ Nobuyuki OhGa, M.D., Yoshio OHNishi, M.D., \\ and Mitsuhiro Yokoyama, M.D.
}

\section{SUMMARY}

To evaluate the significance of the signal-averaged $\mathrm{P}$ wave in various pathological conditions of the atrium, signal-averaged electrocardiograms and echocardiograms were studied in the following 4 groups: (1) 10 normal subjects (control group), (2) 24 patients with paroxysmal atrial fibrillation or flutter (AF) (AF group), (3) 12 patients with left atrial overload without AF (LA group), and (4) 10 patients with right atrial overload without AF (RA group). Original $\mathrm{P}$ wave durations showed no significant difference among the 4 groups. Filtered $P$ wave durations (F-PDs) in the AF and LA groups were significantly longer than that in the control group. F-PD correlated significantly with left atrial dimension (LAD). F-PD in AF patients with LAD shorter than $40 \mathrm{~mm}$ was significantly longer than that in the control group, but there was no significant difference in F-PD between AF patients with LAD longer than $40 \mathrm{~mm}$ and the LA group. Root mean square voltages of original P waves were significantly higher in the LA and RA groups than that in the control group, but in AF patients it did not differ from that in the control group. In conclusion, the signal-averaged $P$ wave is useful to predict atrial fibrillation, but prolongation of F-PD is seen not only in patients with AF but also in patients with LA overload. (Jpn Heart J 34: 29-39, 1993.)

\section{Key Words :}

Atrial fibrillation

Left atrial overload

$P$ wave Signal-averaged electrocardiogram Right atrial overload

$\mathrm{T}$

HE signal-averaged electrocardiogram has been an established method to predict ventricular tachycardia and sudden death. ${ }^{1,2)}$ Late potenJapan.

From the First Department of Internal Medicine, Kobe University, School of Medicine, Kobe,

Mailing address: Hidemi Ogawa, M.D., First Department of Internal Medicine, Kobe University, School of Medicine, Kusunoki-cho, Chuo-ku, Kobe City 650, Japan.

Received for publication January 27, 1992.

Accepted July 1, 1992. 
tials detected by these methods are thought to reflect the slow conduction that favors reentry. ${ }^{3}$ Recently, some studies utilizing signal-averaged electrocardiograms of $\mathrm{P}$ waves to predict paroxysmal atrial fibrillation or flutter (AF) have been reported. ${ }^{4)-8}$. They revealed that the filtered $\mathbf{P}$ wave duration was prolonged in patients with paroxysmal AF and showed the usefulness of the signal-averaged $\mathrm{P}$ wave as a screening test for occurrence of $\mathrm{AF}$. The correlation between the signal-averaged $\mathrm{P}$ wave and electrophysiological evaluation of atrial vulnerability has also been investigated. The result indicated that filtered $\mathrm{P}$ wave duration was prolonged in patients with atrial vulnerability. ${ }^{6}$ But the signal-averaged $P$ wave in various pathological conditions of the atrium without atrial fibrillation has not been well investigated. Prolongation of filtered $\mathrm{P}$ wave duration might be caused only by atrial dilatation or atrial overload.

In this study, we examined the characteristics of $\mathrm{P}$ waves in both filtered and unfiltered form in patients with $\mathrm{AF}$ and in patients with atrial overload without $\mathrm{AF}$, and tried to reevaluate the clinical significance of the signalaveraged $\mathrm{P}$ wave as a predictor of $\mathrm{AF}$.

\section{Methods}

The following normal subjects and 3 patient groups were studied. (1) Control group consisted of 10 normal subjects. (2) Atrial fibrillation or flutter (AF) group consisted of 24 patients in whom paroxysmal atrial fibrillation or flutter was documented at least twice on standard electrocardiograms or 24-hour ambulatory electrocardiographic monitoring, or in whom sinus rhythm was maintained for more than 2 days following cardioversion by antiarrhythmic drugs or $\mathrm{DC}$ shock. The underlying cardiac diseases were hypertrophic cardiomyopathy in 2 patients, mitral regurgitation in 1, effort angina pectoris in 1 , sick sinus syndrome in 1 , and aortic regurgitation in 1. The other 18 patients had no obvious organic cardiac disease. Among the patients with underlying cardiac diseases, left atrial dimensions were less than $40 \mathrm{~mm}$ in 3 patients and more than $40 \mathrm{~mm}$ in 3 . Patients with obvious left atrial overload were not included in this group. (3) The left atrial overload (LA) group consisted of 12 patients without AF with left atrial enlargement due to mitral valve disease. These included mitral valve stenosis in 6 patients and mitral valve regurgitation in 6 patients. Left atrial enlargement was diagnosed if left atrial dimension was longer than $40 \mathrm{~mm}$ by echocardiography. (4) The right atrial overload (RA) group consisted of 10 patients with pulmonary hypertension but without AF. Pulmonary hypertension was diagnosed if pulmonary arterial mean pressure was higher 
than $20 \mathrm{mmHg}$ by cardiac catheterization using a Swan-Ganz catheter. Pulmonary hypertension was due to chronic obstructive pulmonary diseases in these patients. There were 37 males and 19 females with a mean age of 54.2 years, ranging from 23 to 80 years.

After informed consent was obtained, a signal-averaged electrocardiogram was recorded. All antiarrhythmic drugs were discontinued at least 48 hour before the study. Electrocardiograms from body surface bipolar $\mathrm{X}, \mathrm{Y}$, and $\mathrm{Z}$ leads were recorded during sinus rhythm. The $\mathrm{X}$ lead was between left and right mid-axillary lines at the fourth intercostal space. The $Y$ lead was between the right sternal border at the second intercostal space and $\mathrm{V}_{5}$. Between $\mathrm{V}_{2}$ and directly posterior was used as the $\mathrm{Z}$ lead. The electrocardiographic signals containing no atrial premature beats were used for

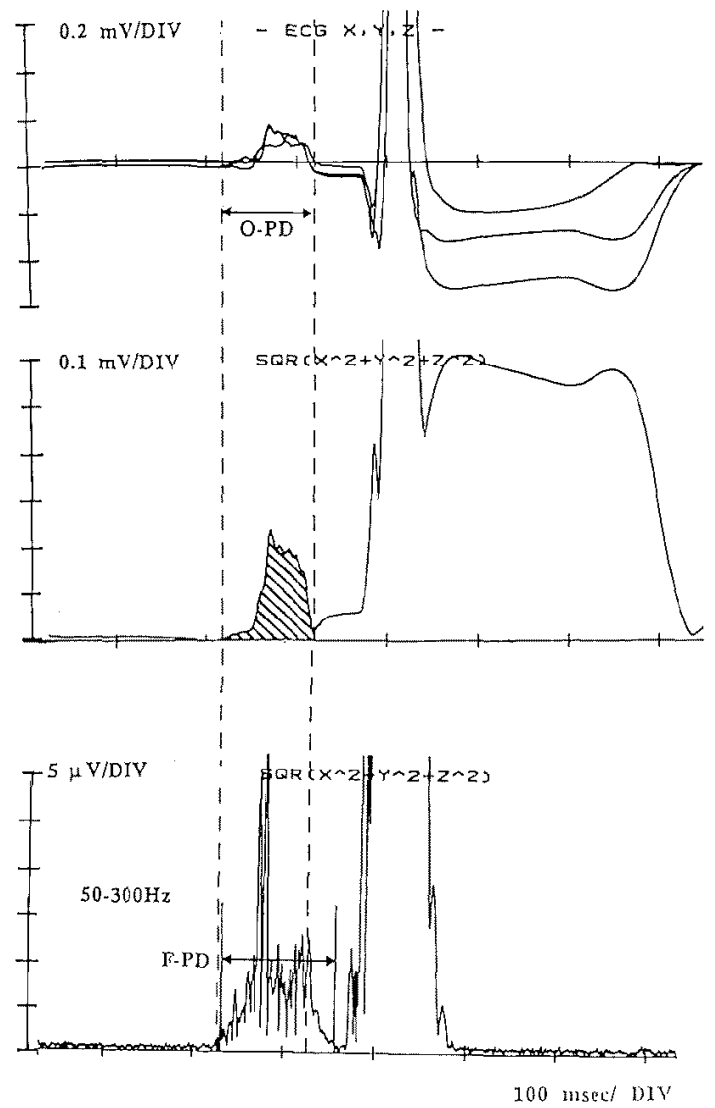

Fig. 1. Measurements of duration of original $P$ wave (O-PD), filtered $\mathrm{P}$ wave (F-PD), and root mean square voltage of original $\mathrm{P}$ wave the area of diagonal lines). The upper panel is $\mathrm{ECG}$ from orthogonal $\mathrm{X}, \mathrm{Y}$, and $\mathrm{Z}$ leads, the middle panel is original EGG combined into a vector magnitude, and the lower panel is filtered ECG combined into a vector magnitude. 
the next procedure. The signals were passed through the band pass filter (NF. FV-604 T; $24 \mathrm{~dB} /$ octave) with a range of 50 to $300 \mathrm{~Hz}$. The nonfiltered and filtered signals were amplified, averaged 250 times with QRS trigger and combined into a vector magnitude with a signal processor (SANEI 7T17). Ventricular premature beats and QRS complexes which did not match a prerecorded template were rejected for analysis. Baseline noise was lower than $1 \mu \mathrm{V}$ and generally lower than $0.5 \mu \mathrm{V}$.

The following quantitative signal-averaged variables were measured as shown in Fig. 1: (1) the duration of the original $\mathrm{P}$ wave (O-PD), that is the interval between the earliest onset and the latest end of original $P$ waves shown in three leads; (2) the duration of the filtered $\mathrm{P}$ wave (F-PD), that is the interval between the onset and the end of filtered $\mathrm{P}$ wave greater than twice the noise level; (3) root mean square voltage (RMSV) of the original $\mathrm{P}$ wave.

Echocardiography was also performed in all subjects studied and left atrial dimension was measured.

Values are presented as mean \pm standard deviation. Unpaired t-test was used for statistical analysis. $P$ values less than 0.05 were considered statistically significant.

\section{Results}

Age, sex, type of arrhythmias, underlying heart diseases, original $\mathrm{P}$ wave durations, and filtered $\mathrm{P}$ wave durations are shown in Table I.

O-PDs were $98.4 \pm 9 \mathrm{msec}$ in the control group, $104.9 \pm 16 \mathrm{msec}$ in the AF group, $106.1 \pm 13 \mathrm{msec}$ in the LA group, and $97.7 \pm 14 \mathrm{msec}$ in the RA group. O-PDs in the AF and LA groups tended to be longer than those in other groups but no significant difference was found among the 4 groups. F-PDs were

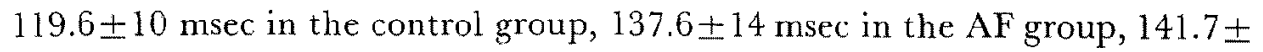
$10 \mathrm{msec}$ in the LA group, and $105.2 \pm 10 \mathrm{msec}$ in the RA group. F-PDs were significantly longer in both the AF and LA groups than that in the control group, but no significant difference was found between the AF and LA groups. F-PD in the RA group was significantly shorter than that in the control group.

Figure 2 shows the correlation between left atrial dimension (LAD) and F-PD in all subjects studied. Significant positive correlation $(\mathbf{R}=$ 0.67) was observed between LAD and F-PD. F-PD seemed to be affected by LAD. To evaluate the significance of the filtered $\mathrm{P}$ wave apart from the influence of LAD, we divided AF group patients into 2 subgroups according to LAD. AF-1 consisted of 15 patients with LAD shorter than $40 \mathrm{~mm}$, and AF-2 consisted of 9 patients with LAD longer than $40 \mathrm{~mm}$. 
Table I. Clinical Characteristics, Original P Wave Duration (O-PD), and Filtered $P$ Wave Duration (F-PD) in 4 Groups

\begin{tabular}{|c|c|c|c|c|}
\hline & Gontrol group & AF group & LA group & RA group \\
\hline $\mathrm{n}$ & 10 & 24 & 12 & 10 \\
\hline age & $28.5 \pm 2$ & $54.9 \pm 17$ & $59.7 \pm 15$ & $68.3 \pm 6$ \\
\hline sex (male) & $10(100 \%)$ & $12(80 \%)$ & $9(38 \%)$ & $6(60 \%)$ \\
\hline \multicolumn{5}{|c|}{ Type of arrhythmia } \\
\hline af & - & $19(80 \%)$ & - & - \\
\hline $\mathrm{AF}$ & - & $2(8 \%)$ & - & - \\
\hline af and AF & - & $3(12 \%)$ & - & - \\
\hline \multicolumn{5}{|c|}{ Underlying disease } \\
\hline MS & 一 & - & $6(50 \%)$ & - \\
\hline MR & - & $1(4 \%)$ & $6(50 \%)$ & - \\
\hline $\mathrm{HCM}$ & - & $2(8 \%)$ & - & - \\
\hline AP & - & $1(4 \%)$ & - & - \\
\hline AR & 一 & $1(4 \%)$ & - & - \\
\hline SSS & 一 & $1(4 \%)$ & - & - \\
\hline COPD & - & - & - & $10(100 \%)$ \\
\hline O-PD (msec) & $98.4 \pm 6$ & $104.9 \pm 16$ & $106.1 \pm 13$ & $97.7 \pm 14$ \\
\hline F-PD (msec) & $119.6 \pm 10$ & $137.6 \pm 14 * *$ & $141.7 \pm 10^{* *}$ & $105.2 \pm 10^{*}$ \\
\hline
\end{tabular}

af =atrial fibrillation; $A F=$ atrial flutter; $M S=$ mitral stenosis; $M R=$ mitral regurgitation; $\mathrm{HCM}=$ hypertrophic cardiomyopathy; $\mathrm{AP}=$ angina pectoris $; \mathrm{AR}=$ aortic regurgitation $; \mathrm{SSS}=$ sick sinus syndrome; $\mathrm{COPD}=$ chronic obstructive pulmonary disease ; $\mathrm{O}-\mathrm{PD}=$ original $\mathrm{P}$ wave duration; $\mathrm{F}-\mathrm{PD}=$ - filtered $\mathbf{P}$ wave duration. $* \mathbf{P}<0.01$, ** $\mathrm{p}<0.001$ vs control group.

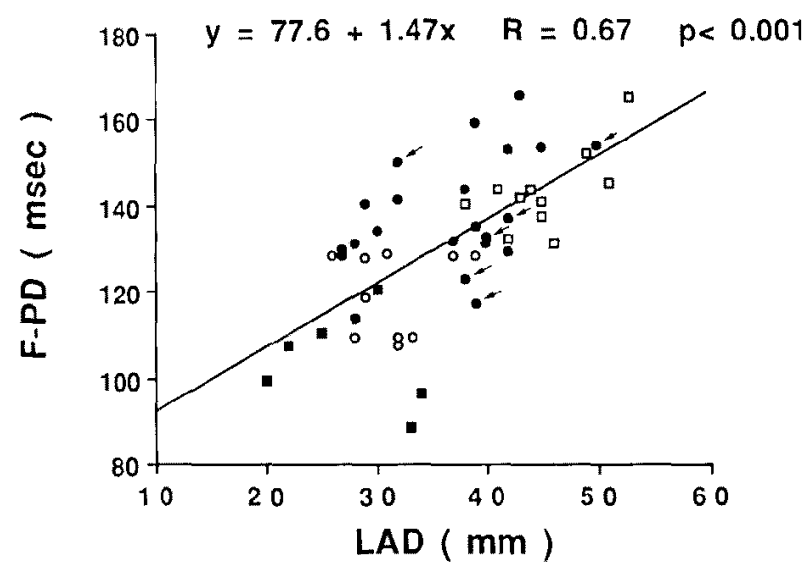

Fig. 2. Correlation between left atrial dimension (LAD) and filtered $P$ wave duration (F-PD) in all subjects studicd. Open circles represent normal subjects, closed circles represent patients in AF group (arrows indicate patients with organic cardiac disease), open squares represent patients in LA group, and closed squares represent patients in RA group. 


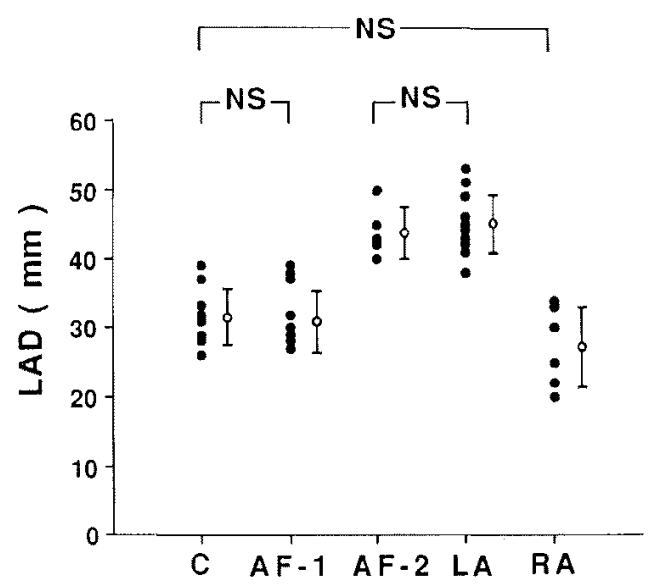

Fig. 3. Left atrial dimension assessed by echocardiogram in each group. $\mathrm{C}=$ control group $; \mathrm{AF}=$ atrial fibrillation or flutter group; $\mathrm{LA}=\mathrm{LA}$ group ; $\mathrm{RA}=\mathrm{RA}$ group ; $\mathrm{NS}=$ not significant.

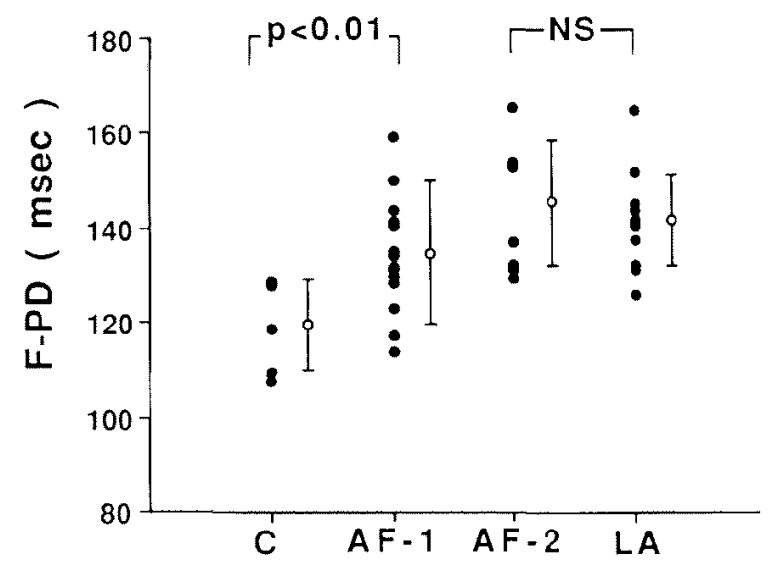

Fig. 4, Filtered $\mathrm{P}$ wave duration in each group. $\mathrm{C}=$ control group; $\mathrm{AF}=$ atrial fibrillation or flutter group $\mathrm{LA}=\mathrm{LA}$ group $\mathrm{NS}=$ not significant.

LADs in the control group, AF-1, AF-2, LA, and RA groups are shown in Fig. 3. There were no significant differences in LADs between the control and AF-1 group, between the AF-2 and LA group, or between the control and RA group. F-PDs in the control, AF-1, AF-2, and LA groups are shown in Fig. 4. F-PD in AF-1 was significantly longer than that in the control group $(134.8 \pm 15 \mathrm{msec}$ vs $119.6 \pm 10 \mathrm{msec}, \mathrm{p}<0.01)$. But no significant difference was found in F-PD between the AF-2 and LA groups (145.5 \pm 13 msec vs $141.7 \pm 10 \mathrm{msec}, \mathrm{NS})$.

RMSVs of the original $P$ wave in 5 groups are shown in Fig. 5. There was no significant difference in RMSV between the control and AF-1 groups 


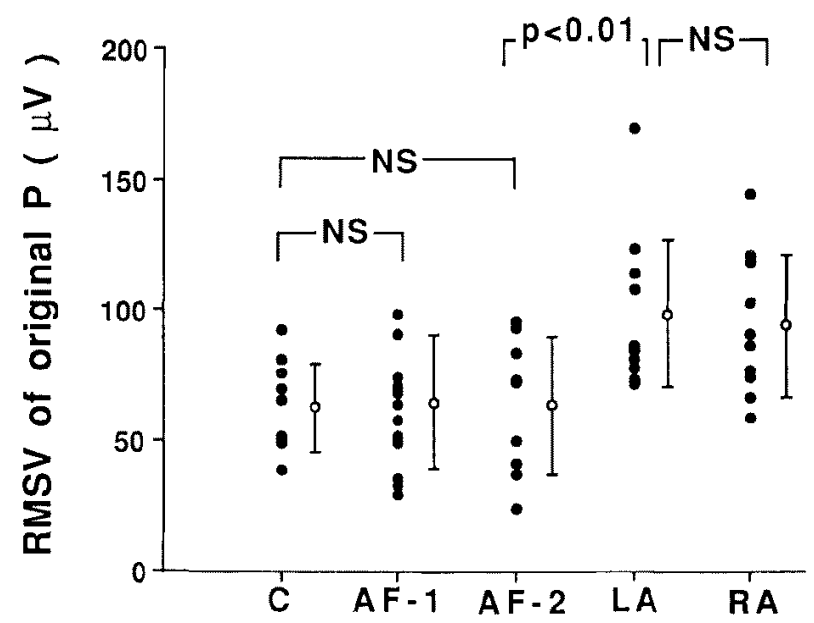

Fig. 5. Root mean square voltage of original $P$ wave in each group. $\mathrm{C}=$ control group $\mathrm{AF}=$ atrial fibrillation or flutter group $\mathrm{LA}=\mathrm{LA}$ group ; RA=RA group; $\mathrm{NS}=$ not significant.

$(62.9 \pm 17 \mu \mathrm{V}$ vs $65.1 \pm 26 \mu \mathrm{V}$, NS). RMSV in AF-2 was significantly lower than that in the LA group $(63.7 \pm 26 \mu \mathrm{V}$ vs $99.1 \pm 28 \mu \mathrm{V}, \mathrm{p}<0.01)$ and did not differ from those in the control and AF-1 groups. No significant difference was found in RMSV between the LA and RA groups $(94.3 \mu \mathrm{V} \pm 27)$.

\section{Discussion}

Allessie et $\mathrm{al}^{91}$ proposed the leading circle hypothesis as a mechanism of atrial fibrillation. Leading circle is a micro reentry without an inexcitable central obstacle and most clinical atrial fibrillations are thought to consist of such multiple micro reentries.

To examine the susceptibility to atrial fibrillation or flutter, several indicators reflecting atrial vulnerability have been reported. Fragmented atrial activity, repetitive atrial firing, short effective refractory period of the atrium, dispersion of atrial refractoriness, and intra-atrial conduction delay have been reported in patients with paroxysmal atrial fibrillation as factors which favor reentry. ${ }^{10)-17}$ However, these indicators could only be identified by electrophysiological study. Therefore, signal-averaged electrocardiogram of the $\mathrm{P}$ wave might be noteworthy as a noninvasive technique to predict atrial fibrillation and flutter.

\section{Significance of filtered $P$ wave}

Prolongation of original $\mathrm{P}$ wave duration is an accepted indicator of 
interatrial conduction disturbances. ${ }^{17}$ ) In this study, original $\mathrm{P}$ wave duration was longer in patients with $\mathrm{AF}$ than that in normal subjects, but the difference was not significant. On the other hand, filtered $\mathrm{P}$ wave duration was significantly longer in patients with AF than that in normal subjects. Therefore, filtered $P$ wave duration seems to be a better marker of conduction disturbance of the diseased atrial muscle than original $\mathrm{P}$ wave duration.

Tanigawa et al ${ }^{18}$ investigated right intraatrial electrograms from 12 points during sinus rhythm in patients with paroxysmal atrial fibrillation. They found more frequently fragmented and wider electrograms in the high, middle, and low atrium in patients with atrial fibrillation than in control patients. Cosio et al ${ }^{16)}$ investigated conduction delay induced by extrastimulation from the high right atrium in patients with paroxysmal atrial fibrillation. They found that the patients had longer conduction delays from the high right atrium both to the His-bundle and to the coronary sinus. These findings indicate that conduction disturbance which favors reentry is widespread in the atrium in patients with paroxysmal atrial fibrillation. Prolongation of filtered $\mathrm{P}$ wave duration in patients with $\mathrm{AF}$ in this study might reflect similar electrophysiological characteristics. Endocardial mapping of the left atrium during sinus rhythm or left atrial extrastimulation is of interest and might reveal such fractionated electrograms.

\section{Filtered $P$ wave duration in atrial overload}

Left atrial size is an important factor to identify patients in sinus rhythm who are at risk of developing atrial fibrillation. Henry et al ${ }^{191}$ reported that atrial fibrillation was common when the left atrial dimension exceeded $40 \mathrm{~mm}$. In this study, patients with left atrial overload, though they had no history of $\mathrm{AF}$, had the same longer filtered $\mathrm{P}$ wave duration as patients with $\mathrm{AF}$. Furthermore, filtered $\mathbf{P}$ wave duration was significantly correlated with left atrial dimension.

To clarify whether filtered $P$ wave duration could be an indicator of $\mathrm{AF}$ in addition to left atrial dimension, we divided patients with $\mathrm{AF}$ into 2 subgroups according to left atrial dimension greater or less than $40 \mathrm{~mm}$. Filtered $\mathrm{P}$ wave duration could differentiate AF patients with left atrial dimension less than $40 \mathrm{~mm}$ from normal subjects. But among patients with left atrial enlargement, it could not differentiate patients with AF from patients with left atrial overload but without AF. Engel et al ${ }^{7)}$ showed no significant difference in filtered $\mathrm{P}$ wave duration between patients with $\mathrm{AF}$ and patients with organic heart disease without $\mathrm{AF}$, and denied the clinical significance of the signal-averaged electrocardiogram of the $P$ wave as an 
indicator of atrial fibrillation. But they did not divide patients according to the left atrial dimension. Prolongation of filtered $P$ wave duration seems to be an indicator of atrial fibrillation among patients with normal left atrial dimension but not a specific finding of atrial fibrillation among patients with atrial dilatation.

In canine experiments, left atrial enlargement has little effect on atrial cell electrophysiology. But histological studies revealed an unusually large amount of connective tissue present between greatly hypertrophied cells. ${ }^{20}$ In patients with left atrial overload, a similar structural change in the atrium could be expected. Such morphologic change might produce conduction disturbance and the prolongation of filtered $\mathrm{P}$ wave duration.

Boyden et $\mathrm{al}^{21)}$ produced right atrial enlargement surgically in canine experiments. They reported that transmembrane action potentials of the right atrium were not different from control, but histological studies showed hypertrophy of muscle fibers and increases in connective tissue between cells. These findings are similar to those observed in canine experiments with left atrial enlargement. Morphologic changes in the right atrium should also be reflected in filtered $P$ waves. But in this study, patients with right atrial overload showed shorter filtered $\mathrm{P}$ wave duration than normal subjects. Filtered $\mathrm{P}$ wave is the sum of right and left atrial electrograms. Therefore, histological damage only in the right atrium did not alter total filtered $\mathrm{P}$ wave duration in this study. Volume overload such as tricuspid regurgitation or congenital heart disease may have different effects on the $\mathbf{P}$ wave. The reason filtered $\mathbf{P}$ wave duration was shorter than in normal subjects was not clear. Underlying disease in the RA group was chronic obstructive pulmonary disease in this study and conditions of the lung or deformity of the thorax should affect surface electrograms. Most of the patients in the RA group showed dyspnea during this study. Therefore, the baseline noise level of the filtered $\mathbf{P}$ wave tended to be higher than that of normal controls even after averaging 250 measurements. That might be one cause of the difference.

RMSV of original $\mathrm{P}$ waves was higher in patients with left and right atrial overload. But RMSV of original $\mathrm{P}$ waves in patients with $\mathrm{AF}$, even in cases with combined left atrial dilatation, was not higher than normal subjects. It is possible that cellular hypertrophy due to atrial overload was reflected by the high voltage of original $\mathrm{P}$ waves. RMSV seemed to be helpful in determining whether the $\mathrm{P}$ wave was affected by atrial overload or not. 


\section{Limitations and clinical implications}

In this study, averaging of $P$ waves was performed with QRS triggering. Thus, filtered $P$ wave duration could be evaluated only on the condition that the P-Q interval is fixed. Patients who showed fluctuation of P-Q interval were not included and premature atrial complexs were excluded from this study. However, $\mathbf{P}$ wave triggering and $\mathbf{P}$ wave template should be better able to obtain more exact values. ${ }^{81}$ The mean age of the AF group was significantly higher than that of the control group. Atrial degeneration caused by aging also affects the $\mathrm{P}$ wave. The effect of aging on filtered $\mathrm{P}$ wave duration should also be investigated.

\section{Conclusion}

Prolongation of filtered $\mathrm{P}$ wave duration seems to reflect conduction disturbances of the atrium, especially the left atrium. Therefore, filtered $\mathrm{P}$ wave duration should be prolonged in various conditions that cause left atrial damage whether the conduction disturbance predisposes patients to atrial fibrillation or not. Filtered $\mathrm{P}$ wave duration should be an indicator of AF among patients with normal left atrial dimension, but in cases of patients with left atrial dilatation, it was not a specific indicator for AF. Left atrial dimension or left atrial overload should be taken into account in evaluating the filtered $P$ wave as an indicator of atrial fibrillation or flutter. Atrial overload seemed to be reflected by the high voltage of original $P$ waves. Evaluation of combined F-PD and RMSV of $P$ waves may be useful to predict atrial fibrillation.

\section{References}

1. Simsom MB: Use of signals in the terminal QRS complex to identify patients with ventricular tachycardia after myocardial infarction. Circulation 64: 235, 1982

2. Ohnishi $\mathrm{Y}$, Inoue $\mathrm{T}$, Fukuzaki $\mathrm{H}$ : Value of the signal-averaged electrocardiogram as a predictor of sudden death in myocardial infarction and dilated cardiomyopathy. Jpn Circ J 54: 127, 1990

3. El-Sherif N, Gomes JAC, Restivo M, Mehra R: Late potentials and arrhythmogenesis. PACE 8: 440, 1985

4. Yamaguchi I, Kuga K, Suzuki H, Sugishita Y, Ito I: The signal-averaged electrocardiogram as a screening test for occurrence of paroxysmal atrial fibrillation (abstr). Jpn Circ J 52: 986, 1988

5. Sagara K, Fu T, Iinuma $H, K$ ato $\mathrm{K}$, Koyama $\mathrm{S}$ : Usefulness of signal averaging Eg to forcknowledge of paroxysmal atrial fibrillation (abstr). Jpn Circ J 52: 986, 1988

6. Kasamaki Y, Ozawa Y, Tanigawa N, Watanabe I, Nagasawa M, Jinno K, Kojima R, Yakubo S, Takahashi Y, Mizobuchi H, Saito S, Hatano M: Paroxysmal atrial fibrillation or flutter-comparison between $P$ wave trigger and $R$ wave trigger (abstr). Jpn Circ J 53: 490, 1989

7. Engel TR, Vallone N, Windle J: Signal-averaged electrocardiograms in patients with atrial 
fibrillation or flutter, Am Heart J 115: 592, 1988

8. Fukunami M, Yamada $T$, Ohmori M, Kumagai K, Umemoto K, Sakai A, Kondoh N, Minamino $\mathrm{T}$, Hoki N: Detection of patients at risk for paroxysmal atrial fibrillation during sinus rhythm by $\mathbf{P}$ wave-triggered signal-averaged electrocardiogram. Circulation 83: 162, 1991

9. Allessie MA, Bonke FIM, Schopman FJG: Circus movement in rabbit atrial muscle as a mechanism of tachycardia. III. The "leading circle" concept; a new model of circus movement in cardiac tissue without the involvement of an anatomical obstacle. Circ Res 41: 9,1977

10. Ohe T, Matsuhisa M, Kamakura S, Yamada J, Sato I, Nakajima K, Shimomura K: Relation between the widening of the fragmented atrial activity zone and atrial fibrillation. Am J Cardiol 52: 1219, 1983

11. Engel TR, Luck JC, Leddy CL, Gonzalez ADC: Diagnostic implications of atrial vulnerability. PACE 2: 208, 1979

12. Niwano S, Aizawa Y: Fragmented atrial activity in patients with transient atrial fibrillation. Am Heart J 121: 62, 1991

13. Boutjdir M, Heuzey JYL, Lavergne T, Chauvaud S, Guize L, Carpentier A, Peronneau P: Inhomogeneity of cellular refractoriness in human atrium: Factor of arrhythmia? PACE 9: 1095,1986

14. Simpson RJ, Amara I, Foster JR, Woelfel A, Gettes LS: Thresholds, refractory periods, and conduction times of the normal and diseased human atrium. Am Heart J 116: 1080, 1988

15. Buxton AE, Waxman HL, Marchlinski FE, Josephson ME: Atrial conduction: Effects of extrastimuli with and without atrial dysrhythmias. Am J Cardiol 54: 755, 1984

16. Cosio FG, Palacios J, Vidal JM, Cocina EG, Gomez-Sanchez MA, Tamargo L: Electrophysiologic studies in atrial fibrillation. Slow conduction of premature impulses: A possible manifestation of the background for reentry. Am J Cardiol 51 : 122, 1983

17. Simpson RJ, Foster JR, Gettes LS: Atrial excitability and conduction in patients with interatrial conduction defects, Am J Cardiol 50: 1331, 1982

18. Tanigawa M, Shimizu A, Matsumoto Y, Sakamoto T, Hashimoto T, Mori M, Kadena M, Ahmed R, Kiya F, Fukatani M, Hashiba K: Electrophysiologic characteristics of right atrial electrograms during sinus rhythm in patients with paroxysmal atrial fibrillation. Jpn Circ J 52: 927, 1988

19. Henry WL, Morganroth J, Pearlman AS, Clark GE, Redwood DR, Itscoitz SB, Epstein SE: Relation between echocardiographically determined left atrial size and atrial fibrillation. Circulation 53: 273, 1976

20. Boyden PA, Tilley LP, Pham TD, Liu SK, Fenoglio JJ, Wit AL: Effects of left atrial enlargement on atrial transmembrane potentials and structure in dogs with mitral valve fibrosis. Am J Cardiol 49: 1896, 1982

21. Boyden PA, Hoffman BF: The effects on atrial electrophysiology and structure of surgically induced right atrial enlargement in dogs. Circ Res 49: 1319, 1981 